\title{
Communicating for survival in the mining and construction industries: Northern
}

\author{
conversations and Southern contextualisations
}

\section{ABSTRACT}

The Global South, as the collective for the peripheries of mainstream development is known, is often regarded as merely a beneficiary of Northern borne notions and theories in the field of organisational communication. The problem is that the Southern context and circumstance does not always mirror that of the North, which means that these dominant, revered theories are not necessarily applicable. One Southern context is that of the South African mining and construction industries which is seen as notoriously dangerous, plagued by various obstacles to internal organisational communication (such as illiteracy and diversity) and what Le Roux and Naudé (2009:29) refer to as "historical baggage". The research question of this paper is hence whether congenital Northern communication theories can be adequately incorporated into the unique Global South in order to fulfil the important task of communicating safety information to employees. The article explores the appropriate implementation of the principles of the excellence theory, the stakeholder theory as well as the relationship management theory and the research methodology includes interviews, focus groups and quantitative questionnaires at two organisations. The result of the empirical research is the amalgamation and reworking of these theories' principles into a model for internal safety communication applicable to the South. 


\section{INTRODUCTION}

In a special edition of Communicatio (issue 2 of 2012) on the issue of Africanisation and communication theory as it endeavoured to investigate the development of an African theory of communication, the editor admitted that this has "not yet been fully answered [to] and still requires[s] further exploration" (Du Plessis 2012, 124). Ostensibly, an African theory of communication would seek to be specifically suited to 'Africa' as it developed from 'Africa'. The problem is that Africa in itself is exceedingly diverse, proffering an almost infinite array of communication contexts. One man's 'Africa' could differ from another man's 'Africa' as much as it would from any Western or Northern context.

Although the development of a single African theory of communication might be perceived as ambitious, the debate the edition ventured to incite is valuable and de rigueur for the field of communication to address the questions of "whether Africa has a unique contribution to make to communication theory";and "whether there is something uniquely African that could contribute to our understanding of communication" (Du Plessis 2012, 123)

A remnant of colonialism, Bourgault $(1995,2)$ avers, is that Africa has been treated as tabula rasa by communication scholars; as a 'clean slate' absent of premeditated ideas or notions, ready to absorb those from a Northern paradigm and implement them unswervingly. African scholars themselves have perhaps contributed to the continuation of this legacy within the organisational communication field by holding forth 'Northern notions' as revered and established epistemologies; teaching and declaiming theoretical models from this context to be (if anything) only relatively imperceptibly adapted to the Southern circumstance. The main problem is that the Southern context often does not mirror the North which means that its unique circumstances dictate a different handling. Northern notions are not strictly transferable or even translatable, although timehonoured internal organisational theory is mostly consequential of Northern notions. The unique circumstances of the mining and construction industries in South Africa, as 
representative of the Southern context, are simultaneously credited with being the backbone of the country's economy and responsible for the most work-related deaths in its employee population due to inherent hazardous working conditions (Davenpoort 2005). Furthermore, internal organisational communication in these industries can be classified as inimitable due to the unique nature of its workforce, which is described as being exceptionally diverse (Greeff 2011; Le Roux and Naudé 2009). Internal organisational theory mostly consequential of Northern notions would, therefore not necessarily be applicable to this context. Rather than the established nature of the theory being the basis for its implementation, the context of the communication should dictate its execution.

The main aim of this article is to empirically explore this statement by considering the ways in which the context of communication can change and further the communication theory applied to it

\section{CONTEXTUALISATION}

The study is contextualised within the context of the mining and construction industries of South Africa where two major aspects affect the unique nature of the Southern context which necessitates the modification of Northern notions: the importance of safety communication due to hazardous working conditions; and the unique nature of the employee body.

\section{The importance of safety communication}

In addition to being an economic strength of South Africa, the mining and construction industries are responsible for the highest employee mortality rate in the country because the working conditions in both industries inherently imply hazardous conditions (Davenpoort 2005). In the earlier days of these industries, as late as the late 1900s, the safety of workers in these hazardous conditions was not regarded as earnestly as it could have been - an aspect that has changed almost farcically. 
One of the reasons for this discernment transferral is the pressure that organisations receive from governing bodies in this country, namely the Department of Labour for the construction industry and the Department of Mineral Resources (previously the Department of Minerals and Energy) for the mining industry. In 1993 and 1996 respectively, the Occupational Health and Safety and the Mine Health and Safety Acts were published, which allow these governing bodies to enforce respectable safety standards in these industries. These standards have to be upheld in an organisation to operate within South Africa by receiving working permits and mineral rights from these governing bodies.

Safety in organisations within the mining and construction industries has become an important aspect because these governing bodies hold the power to shut down the operations of an organisation if it is not in line with the prodigious benchmark set. For example, Thabo Gazi, the Chief Inspector of Mines, said: "government is determined to ensure that safety measures in South Africa ... are improved and we will not compromise on achieving that objective" (Prinsloo 2010, 1).

Another measure that these acts implement to ensure that safety in organisations is observed and its standards upheld is the employment of 'competent persons' based on their safety qualifications and experience who are responsible for the safety in the organisation. In terms of the focus of this study, it is argued that safety communication in organisations within the mining and construction industries is functioned by individuals trained in safety, not internal organisational communicator.

\section{The unique nature of the employee body}

In addition to the general diversity of the population of South Africa, the body of employees in these industries are diverse and unique in terms of four factors: origin, culture, language and literacy (Greeff 2011; Le Roux and Naudé 2009; Creamer 2002).

The influx of workers from South Africa, southern Africa and Africa as a whole, resulted in diversity in the employee body in terms of origin, especially in terms of geographical 
origins due to the presence of a migrant workforce. Migrant workers are workers not originally from the area where they work but temporarily live close to the operation where they work, not permanently with their families, and usually go home on 'pay weekends' in order to deliver money to their families (Meekers 2000, 21). The derivation of the phenomenon of a migrating workforce is traced back to the inception of the mining and construction industries through an influx of individuals driven to towns where mining and construction operations were booming in search of employment and the lucrative nature thereof.

The different geographical origins means migrant workers are also culturally diverse, which in an organisational setting is important because the communication process is one of interpretation (Lustig and Koester 2003, 12) where the reception of the communication message is subsequent to interpretation from the receiver, which is filtered through that receiver's cultural backcloth (DeVito 2009, 34; Lustig and Koester $2003,12)$. The same message can be interpreted in as many different ways as there are cultures at the organisation because a communication message that might "work in one culture might not necessarily work in another" (DeVito 2009, 34). .

The cultural differences also result in the interaction of different languages which can cause confusion or misunderstanding in organisations in these industries, epitomised by the use of the mining language Fanakalo (also spelt Fanagalo). Fanakalo is a mainly Zulu-based pidgin language developed to promote ease of communication by mixing all the various languages spoken in the mines into one (Naudé and Le Roux 2005, 6; Githiora 2002, 164) and spilled over into the construction industry where migrant workers started to settle (Githiora 2002, 164). Although this language once featured as the lingua franca of the mining and construction industries, it has since been branded as racist, with English being declared as the official vernacular. However, in practice Fanakalo in many instances stays the de facto language (Greeff 2011, 118).

Furthermore,these industries offer various employment opportunities for unschooled and illiterate workers and the Leon Commission for Health and Safety reports that 84\% 
of general labourers and machine operators in these industries are functionally illiterate (Creamer 2002, 2; Leon 1995, 70). This means that the internal organisational communication channels used by organisations in these industries need to be mindful of this literacy diversity and should ensure that all employees, regardless of their literacy level, receive and understand the safety communication message. According to Greeff (2011), this implies that many channels traditionally suited to the communication of safety-related messages are rendered useless for a large majority of the mining and construction workforce.

Hence it is argued that internal organisational communication, specifically internal safety communication in these industries, is important because the employee corps do not speak the same language as their diversity intensifies an already arduous context which could result in life and death situations. This offers various obstacles to internal organisational communication and the implementation of pertinent internal organisational communication theory.

\section{THEORETICAL BACKGROUND}

For the purposes of this study, four theories that shaped the current understanding of internal organisational communication from the Northern contextual mindset to internal organisational communication are discussed, namely the stakeholder theory, the relationship management theory, the excellence theory and satisfaction literature. .

From an organisational communication point of view, the stakeholder theory focuses on the impact of relating with stakeholders where employees are seen as one of the most important on the organisation's goal attainment and on its bottom line (Jensen 2010, 33; Angle, Donaldson, Freeman, Jensen and Wood 2008, 154; Freeman, Wicks and Parmar 2004, 365).This viewpoint highlights the importance of employees as stakeholders related to the organisation and emphasises the fact that a relationship should exist between the parties. The main critique against this theory is that it did not, 
in a normative or descriptive way, delineate what this relationship should or does look like in practice.

The next chronological theoretical progression focusing on this relationship is the relationship management theory which describes the organisation-employee relationship from the paradigmatic view offered by the stakeholder theory. This theory identifies those measures of a relationship that exist and are important to underline, as it decisively notes that it is through internal organisational communication that this relationship is initiated and managed (Grunig and Hon 1999, 3; Dozier, Grunig and Grunig 1995, vii). The main critique against this theory is that it does not delineate what communication should look like in this relationship.

The excellence theory proposes to breach this void by addressing internal organisational communication and discussing the parameters whereby it can be classified as excellent or not. It identifies measureable and concrete communication aspects that gauge the excellence of this form of communication (Grunig and Hon 1999, 3; Dozier et al 1995, vii). The excellence theory, as in the case of the stakeholder and relationship management theories, considered the association between the organisation and its employees decidedly from the organisation's perspective in terms of responsibility and recompense. The limitation was that it did not provide a complete view of internal organisational communication from employee perspectives.

The last theoretical advancement is satisfaction literature which looks at employees' perspectives in terms of internal organisational communication. As in the case of the excellence theory, this literature presents measureable and examinable outputs of communication, but this time it is focused on employees' perceptions rather than organisational endeavours (Downs and Hazen 1977, 64).

Applied in practice, the combination of these theories thus offer different progressions and vantage points from which to view internal organisational communication. 
Relating the fundamental assertions or theoretical statements derived from these notable theories to the circumstance of the South African mining and construction industries would firstly see statements of the stakeholder theory include the concept of wholeness, or holism - a remnant of its origins from the systems theory (Jensen 2010, 33; Angle et al 2008, 154). Herein, organisations need to realise that the manner in which they regard or act on employees' need for safety would impact on all operations within this organisation (Angle et al 2008, 154; Freeman et al 2004, 365). Furthermore, the relationship management theory fundamentally revolves around the assertion that the cornerstone of any stakeholder relationship is communication. It is argued that a good relationship instigated by this communication would see employees being more likely to align themselves to the organisational safety objectives, and less likely to interfere with their realisation, for example through safety strikes and litigation, which continually occur in these industries. For the organisation to enjoy this kind of relationship, it would be important that employees have relatively full disclosure of safety information from the organisation and access to the organisation through management, as the other party to the relationship. The power balance in the organisation-employee relationship should also be relatively fair or reasonable and trust, commitment and satisfaction should be present therein (Grunig and Hon 1999, 3; Dozier et al 1995, vii).

Underlying this is the fact that the organisation-employee relationship should be practised in a strategic manner, as proposed by the fundamental assertion of the excellence theory. Inherently, the internal safety communication of any organisation in the mining and construction industries of South Africa should not only be driven by technical communicational aspects, but also make use of symmetrical, two-way flow of communication. This means that a representative for internal safety communication should be part of the dominant coalition of the organisation to ensure the symmetrical two-way nature of it entices a participative culture, equal opportunity for diversity and an organic organisational structure, as far as possible (Downs et al 2004, 57; Grunig and Hon 1999, 3; Dozier et al 1995, vii). 
Similarly, the first fundamental assertion derived from communication satisfaction proposes that the communication climate in an organisation should reflect the goals and objectives of the organisation, with the practice of safety at the proverbial 'factory floor' not contradicting the strategic intent of the organisation. The integration of all departments in the organisation is hence needed to ensure that objectives are met. Furthermore, personal integration of employees as individuals into the main objective of the organisation should also be done where employees should continually be kept up to date on all safety aspects in the organisation in the general sense, as well as in terms of their personal impact on these aspects (Downs and Hazen 1977, 64). External events that impact on the safety in the organisation should be communicated, because understood that employees would feel more integrated if they are simultaneously aware of both their individual places in the practice of safety in the organisation and the overall functioning thereof (Downs and Hazen 1977, 64).

It is, however, argued that the outputs or theoretical statements of these theories are seated within Northern contextual approaches. In order to understand the pertinence to the unique context presented in the South African mining and construction industries, these theoretical statements were assessed empirically within the Southern context.

\section{RESEARCH METHOD}

To adapt or amend the theoretical statements from these four internal organisational theories to the context of the mining and construction industries of South Africa and the communication of safety information internally to employees, these statements were tested in practice. Empirically, this was done by means of three data-gathering methods namely interviews, focus groups and questionnaires at two organisations both seated within the mining as well as the construction industries of South Africa. The first organisation is the Gautrain project, under the management of Bombela Civil Joint Venture and the second Diesel Power Opencast Mining. The appropriateness of selecting these two organisations lies in the fact that the Gautrain project, notwithstanding its mining operations (for example underground tunnelling), is classified 
mainly under the construction industry as DPOM, and its construction operations (for example piling) is classified mainly under the mining industry. As indicated before, the safety systems employed in both these organisations have to adhere to the Mine Health and Safety Act 1996 (No. 29 of 1996) and the Occupational Health and Safety Act (No. 85 of 1993), and are accountable to both the Department of Mineral Resources and the Department of Labour.

Due to the costs to company and the potential impact on production, sampling was utilised. With respect to the Gautrain project (which is a large project in monetary terms and human capital), it was impossible to research all operations based on the specifications of the management. Therefore, for this study, the Precast Yard was specifically selected as an operation. This selection was based on the principles of the convenience sampling method (David and Sutton 2004, 151; Keyton 2006, 126). When reference is made to the Gautrain project in this article, it should thus be understood that it is merely a way of distinguishing between the two organisations used in this research and that it does not presume to make generalisations to this effect.

In terms of Diesel Power Opencast Mining (DPOM), a much smaller operation than the Gautrain project, access was granted by the organisational management to all opencast mining sites, with the exclusion of strictly engineering workshops and construction sites. As a result, seven opencast mining sites (stretching from Gauteng to Mpumalanga) were included in the research. With reference to the selection of the employees to take part in the research, each one of the data-gathering methods had its own population and sampling.

Interviews were conducted with all managers in both organisations who are responsible for internal safety communication (which resulted in three at the Gautrain project and one at DPOM). Interviews were the first empirical research methods employed and focussed on exploring, from the perception of these managers, the appropriateness and adaptation needed to the theoretical statements on the communication of safety information in the mining and construction industries of South Africa. 
Based on the results of these interviews, adaptations to the theoretical statements were made for alignment to the context of the South African mining and construction industries. Following these adaptations, these theoretical statements were formulated into quantitative questionnaire items tested in the general employee populace of each organisation. The questionnaires administered in this research therefore tested the validity of the adapted theoretical concepts and constructs statistically. This was done through calculations to determine the Cronbach's alpha coefficient of every concept's constructs, as well as factor analyses yielded by each. In this way, it could be deduced from the questionnaires to what degree the concepts derived from the theoretical statements of each of the internal organisational communication theories correlate and group with overarching constructs as identified in the literature. To facilitate this, the questionnaire was administered to employees at both organisations, which was preempted by a pilot study with an expert panel with experts in both internal safety communication in the mining and construction industries as well as in the field of internal organisational communication with knowledge of the theories under investigation.

Systematic sampling was used and of the 807 employees of the Gautrain project's Precast Yard a response rate of 281 was achieved, which gave the sample a confidence level of over 95\%, with a satisfactory sampling error of under 5\% (Keyton $2006,122)$. At DPOM access to a sample of 10 employees per site was allowed by management and these employees could be selected without bias as long as the number did not exceed 10 per site, which culminated in a response rate of 73 .

After the administration of the questionnaire at both organisations, focus groups with employees at the Gautrain project were held. The focus group centred on discussing the nature of safety and internal safety communication in the mining and construction industries of South Africa, specifically from the viewpoints of the general employees who work at the proverbial (and sometimes literal) coal face. Three focus group discussions were held with participants: the first focus group consisted of male 
employees of all post levels up to supervisors; the second focus group consisted of male employees on supervisory and higher levels, and the third focus group consisted of female employees of all post levels. Due to the fact that there were few female employees, they were all accommodated in one focus group and not split according to post level.

The focus group participants were selected by means of volunteer sampling: participants who completed the questionnaire were asked whether they were interested in being part of a focus group. The participants showed a keen interest in the topic under investigation, and were willing to take part in the study (Keyton 2006, 127). These participants gave insight into the context of the mining and construction industries of South Africa, and the appropriateness (or not) of the four internal organisational communication theories related to the Southern context.

Based on the results of the focus groups, interviews and questionnaires, inferences regarding the appropriate use of these theories were made to illustrate and underline the robustness of the application and adaptable disposition thereof and the apposite application to the communication of safety information to the diverse internal audience of employees in the mining and construction industries. The findings are discussed in the next section.

\section{FINDINGS}

At the onset it was stated that this research is cognisant of the thrust towards recognising "non-Western and indigenous epistemologies" (Rao and Wasserman 2007:30), as it simultaneously heeds the caution of Nyamnjoh (2011:25) of "losing sight of the imperative of speaking to people close to home" by ignoring Northern ideas and theories. In this research the appropriateness of Northern theories were tested in one of the most unique circumstances found in South Africa - that of its mining and construction industries. Aligned to this notion which underscored this research, the findings obtained from the empirical exploration posit that although substantial adaption 
is needed in many instances regarding these theories, most assertions fundamental to the theories are relevant.

In applying the fundamental theoretical assertions of the four germane theories discussed to the context of the mining and construction industries of South Africa, it became clear that - in order to hold a holistic view of it - the germane assertions from all four theories should be used in concert with one another to ensure the successful communication of safety information in this context.

Based on the empirical findings, these fundamental assertions from the literature could be reformulated as theoretical statements of internal safety communication in the Southern context. These assertions have been grouped into new general themes derived from the interviews and focus groups in terms of its importance in this context, and with the specific focus of internal safety communication. The newly derived themes were categorised into holistic, turbulent, strategic, relational and symmetrical communication and were used as the foundation for the formulation of the model for internal safety communication. The broader themes of the model were labelled as internal safety communication factors, while each consequential lesser theme or grouping was labelled an element of the factor. As indicated above, these factors should be considered holistically, where each one influences and works together with the others. Hence the model is represented circularly, as it is argued that not one of these factors can be separated from the other, is regarded as more important than the other, or should be executed in any order or hierarchy. In applying this model in practice, it should be realised that all elements or factors should be weighted the same and that its functioning should be seen in line with the wholeness principle where the absence of one element influences all others and the whole they create.

Figure 1 indicates the theoretical statements for internal safety communication in the mining and construction industries of South Africa based on the research findings of the interviews and focus groups and were ultimately grouped in terms of 16 elements that constitute five factors of internal safety communication. 


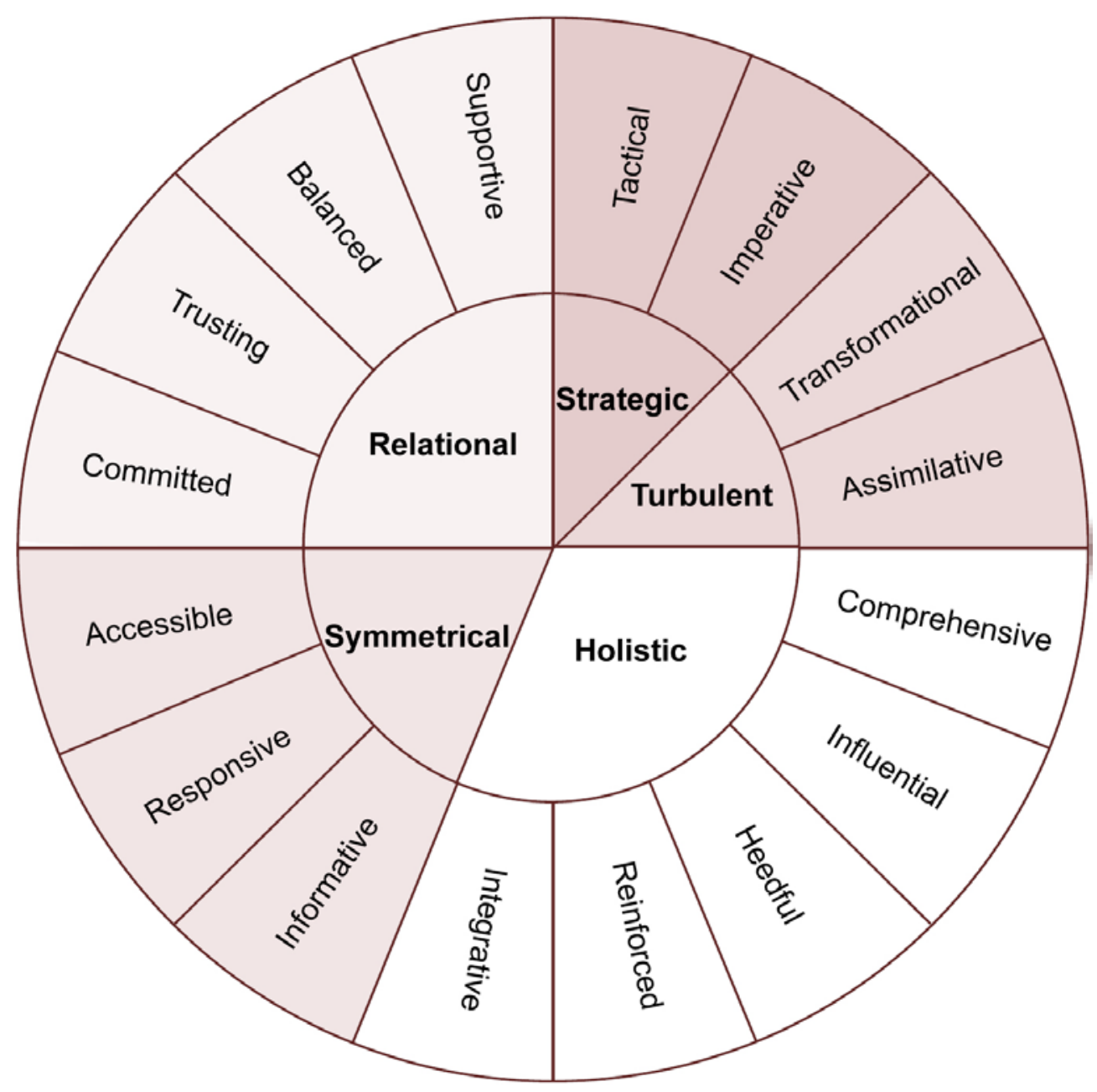

Figure 1: Model for internal safety communication

Holistic internal safety communication, depicted in the lower right-hand side of the model, is mainly derived from the assertions and theoretical statements of the stakeholder theory. The holistic factor addresses the functioning of internal safety communication in terms of its own consideration along with its relation to other organisational functions. This function, due to its encompassing nature, harbours five elements - pointing to the fact that internal safety communication from a holistic perspective should be comprehensive, influential, heedful, reinforced as well as integrative. 
In terms of being comprehensive, it is understood from the findings of the focus groups and interviews that internal safety communication in this context is influenced by many other factors in the organisation's functioning and that a veritable view of internal safety communication is only held when this function is viewed with other functions. This interrelative nature sees internal safety communication as influential through integrative and self-assertive tendencies. In terms of integrative tendencies, internal safety communication would arguably be prone to adapt to other factors; whilst self-assertive tendencies would influence or impact on other facets where the tendency takes credence in any given situation as a matter of hierarchy and previously defined goals. It is hence argued that if the internal safety communication is higher in the organisational hierarchy than an opposing aspect, that it will influence that aspect, or if lower, be influenced by that aspect. If, however, previously mutually defined goals exist in the organisation, both systems yield to those goals rather than to each other.

Another finding which should be aligned with this is heedful internal safety communication which evaluates decisions in terms of the value that is created or destroyed by it. Heedful of employees' safety needs and aligned to the internal safety communication objectives, decisions that counteract the objective of heeding stakeholders' safety needs should be rejected or altered. Hence decisions in the organisation in terms of safety should be reinforced and substantiated by actuality, where the climate of the organisation experienced by employees should reflect the strategic goals and objectives of the organisation, where the perceived importance of safety not contradicting the strategic intent. This emphasises the importance of the cliché that the organisation should not only 'talk the talk' of internal safety communication, but should 'walk the walk'.

Lastly, in terms of its holistic nature, it was indicated that internal safety communication should be integrative on two levels, a functional and an individual level, which is in support of the theoretical statements posed based on satisfaction literature. Functional level integration involves cross-functional and cross-sectional integration between facets, sections and functions of the organisation. Due to the fact that safety in the 
mining and construction industries is a matter of regulation through the country's laws, cross-functional and cross-sectional integration of safety and internal safety communication objectives were seen to be important. It was also indicated that it should not only be the responsibility of safety personnel to enforce and further the realisation of safety objectives, but of all functions and sections within the organisation. On an individual level individuals should, by means of internal safety communication, be made aware of their place and contribution towards the organisation's overarching safety objectives and successes. It was found that if individuals are integrated in this way, for example by means of personalised feedback, they will arguably accept more responsibility and accountability for their own safety involvement or input in the organisation.

The second main factor in the model for internal safety communication in Figure 1 is the turbulence factor, mostly gleaned from the excellence theory. The factor of turbulent internal safety communication builds on the theoretical arguments of the holistic factor, wherein the reactions of internal safety communication to the ever-changing organisational communication environment in which it functions were considered. It was clear from the first factor that internal safety communication should adapt to its environment in terms of its transformational and assimilative prospects. The focus group participants indicated that for transformational internal safety communication, turbulent environments with activist groups should be encouraged, as this kind of turbulence excels communication to better itself to respond to this turbulence. They also indicated that transformational turbulence in an organisational environment could excel internal safety communication beyond the equilibrium it experienced before the turbulence originated. The theoretical statement that turbulence could give way to assimilation of previous diverse perceptions or beliefs between the organisation and its employees, was hence supported. It was further indicated that assimilative internal safety communication should work towards jointly sharing and solving problems brought on by turbulent environments, working cohesively, rather than disjointedly. 
The third factor, strategic internal safety communication, points to the theoretical statement on the purposefulness of this kind of communication in the sense that is it practiced intentional and considered. From the results, it became clear that internal safety communication in these industries is in practice headed and implemented by safety personnel who are not necessarily competent or even equipped communicators. Consequently, it was realised that internal safety communication holds the risk of being run only on a technical or baseline practical level which means that internal safety communication can become disconnected, operating singularly in compartmentalised actions that do not link up with one another or with the strategies and objectives of the organisation. The interview participants realised and indicated that internal safety communication should rather be strategic and purposeful in nature, encompassing the two elements of tactical and interpretive communication. In this regard, the tactical nature of internal safety communication positions that it operates only on a technical level. Although the technical aspects of internal safety communication were regarded as important by the interview participants, it was stressed that this communication should be driven by the mission, goals and objectives of the organisation generally and the safety department specifically. It was also positioned that each aspect or activity should be directly traceable to a specific objective(s) or goal(s). In order to equip this, they indicated that internal safety communication should be imperative in nature, where such communication should form part of the considerations of the dominant coalition in the organisation to allow the formulation and advancement of safety objectives and goals within the organisation.

The relational factor reiterated that the organisation should strive towards building a relationship with its employees through internal safety communication. Based on the theoretical statements of the relationship management theory, it was indicated by the focus group participants that although the general organisation-employee relationship should be established by means of internal communication in any given organisation, a specific relationship in terms of employee safety should also be present. This relationship will impact on and be impacted upon by the general organisation-employee relationship, but should still be seen as important for safety personnel to establish a 
facet within this general relationship that is safety-driven. They indicated that even if the general organisation-employee relationship is not successful, employees should still feel that the organisation is concerned, can be trusted and is committed to their safety, in other words that the overall experience should be that the organisation has a good safety relationship with employees. To this end, the safety relationship in an organisation should be supportive, balanced, trusting and committed.

Lastly, based on the excellence theory and satisfaction literature, it was argued that internal safety communication should be symmetrical in nature, which refers to the fact that internal safety communication should be holistic and balanced in its own right. This links to the factor of strategic communication and include three elements, namely: accessible, responsive and informative safety communication. Based on the empirical findings, it was indicated that accessible internal safety communication should be open to promote participation and full disclosure to endorse participative cultures wherein all employees are open to make suggestions, take part in joint decision-making and are empowered through communication and the sharing of information to contribute towards the reaching of organisational goals in an equitable environment that promotes teamwork. Keeping within participative organisational cultures, the input and feedback generated from employees due to the accessible nature of internal safety communication should be responded to or taken into account by the organisation in order to be responsive. The focus group participants also indicated that the content generated from the feedback of internal safety communication in its accessible element should be interpreted, taken into account, and where applicable, acted upon in the organisation. At the rudimentary level it was indicated in the interviews that internal safety communication should offer guidance to employees as to how to do their job safely, and as indicated in the integrative element, this should be directed and relevant to each employee and his/her specific job situation. The interview participants also indicated that if the internal safety communication is related to relevant guidance on safe work procedure, it should be informative as it should be responsive and accessible, supporting the theoretical statement that internal safety communication will only be successful once the organisation is open and holistic. 
In order to explore the appropriateness of these new groupings of theoretical statements, quantitative measures were implemented. The factors discussed above were regarded as constructs and its elements as concepts. In order to statistically measure the validity and reliability of these groupings these elements were transformed into items and administered as a quantitative questionnaire to employees at the two organisations that took part in the empirical research of this study.

For reliability, the constructs were tested by determining the combined Cronbach's alpha value of its concepts. Table 1 below summarises the Cronbach's alpha value of each of the concepts at the two administrations (the first at the Gautrain project and the second at DPOM).

Table 1: Cronbach's alpha values for concepts and constructs

\begin{tabular}{|l|l|r|r|}
\hline \multirow{3}{*}{ Construct } & Concept & $\begin{array}{c}\text { Cronbach's } \\
\text { alpha in first } \\
\text { measurement }\end{array}$ & $\begin{array}{c}\text { Cronbach's } \\
\text { alpha in second } \\
\text { measurement }\end{array}$ \\
\hline \multirow{4}{*}{ Holistic } & Comprehensive & 0.886 & 0.888 \\
\cline { 2 - 4 } & Influential & -- & 0.758 \\
\cline { 2 - 4 } & Heedful & 0.724 & 0.899 \\
\cline { 2 - 4 } & Reinforced & 0.811 & 0.825 \\
\cline { 2 - 4 } & Integrative & 0.768 & 0.892 \\
\hline \hline \multirow{3}{*}{ Turbulent } & Assimilative & 0.720 & 0.802 \\
\cline { 2 - 4 } & Transformational & 0.814 & 0.801 \\
\hline \hline \multirow{3}{*}{ Strategic } & Imperative & 0.648 & 0.834 \\
\cline { 2 - 4 } & Tactical & 0.698 & 0.699 \\
\hline \hline \multirow{3}{*}{ Symmetational } & Supportive & 0.764 & 0.921 \\
\cline { 2 - 4 } & Balanced & 0.816 & 0.902 \\
\cline { 2 - 4 } & Trusting & 0.860 & 0.920 \\
\cline { 2 - 4 } & Committed & 0.742 & 0.856 \\
\hline \hline & Accessible & 0.817 & 0.861 \\
\cline { 2 - 4 } & Responsive & 0.861 & 0.839 \\
\cline { 2 - 4 } & Informative & 0.844 & 0.832 \\
\hline
\end{tabular}

As indicated in Table 1, all the values are above the acceptable benchmark ( $\geq 0.6)$ with many in the range of excellence ( $\geq 0.8$ ) (David and Sutton 2004, 171), pointing to the 
fact that this is a reliable measure of these concepts. In terms of the groupings of these items, explorative factor analysis found the concepts, as grouped above, validly measuring the constructs they pertain to. In this, meaningful correlations were found in each instance, all above the benchmark of 0.4 (Field 2009:35), ranging between 0.408 and 0.888. Likewise, all factor loadings (even where just one underlying factor was found) were significant, ranging between 0.626 and 0.958 .

It can be inferred from these statistical measures that the 'new' groupings of theoretical statements for internal safety communication can be regarded as valid because employees working in the context of these industries grouped the items as they pertained to the concepts with the constructs they deemed significantly and meaningfully. The results of the respondents on the questionnaires indicated that the adaptation of the four seminal theories of internal organisational communication could be regarded as successful to the specific focus of internal safety communication within these industries of South Africa.

\section{CONCLUSION}

This article explored two aspects: firstly, the appropriateness of the application of Northern notions to the Southern context to indicate its suitability for explaining Southern communication phenomena; and secondly a contest against the notion that the South is tabula rasa which addressed the contribution that the South can make to furthering notions which originated in the North.

The findings pertained to, and supported the exploration of the theoretical statements derived from the four seminal internal organisational theories, namely the stakeholder, relationship management, excellence theories and satisfaction literature which were applied to the unique circumstance of the South African mining and construction industries. It became clear that appropriate application of Northern notions is indeed possible, but that this application should follow from a contextual perspective, where the context takes credence in decisions regarding application, rather than the perceived 
prominence of appraised Northern notions. Once this is achieved, contributions from the Southern circumstance can be made to contribute to the expansion of Northern notions and its application to avenues not previously anticipated or explored. Subsequently based on the findings of the research, this article proposed a new model for internal safety communication for the mining and construction industries which furthers Northern notions and theory into a new approach and application of germane theoretical foundations to the emergence of new theory from the Southern circumstance. This new model challenges the notion of South as tabula rasa and points to the expansion of the field of organisational communication resultant from contexts and acumen solely found in Southern circumstance. Herein the context that engendered this model did indeed "provide for alternative ways of understanding and explaining communication, thereby extending our current theoretical frameworks (without substituting or replacing existing theory, yet while furthering our understanding ...)" (Du Plessis 2012, 124). 


\section{LIST OF SOURCES}

Angle, B.R., T. Donaldson, R.E. Freeman, M.C. Jensen, R.K. Mitchell, and D.J. Wood. 2008. Dialogue: Toward superior stakeholder theory. Business ethics quarterly 18(2):153-190.

Bourgault, L.M. 1995. Mass Media in Sub-Saharan Africa. Bloomington: Indiana University Press.

Creamer, M. 2002. Don't neglect South Africa, warns union. Mining weekly 21 May. http://www.miningweekly.co.za/article.php?a_id=25344 (accessed 21 February 2007).

Davenport, J. 2005. Skills dearth will ultimately restrict consulting industry. http://www.miningweekly.co.za/article.php?a_id=65585 (accessed 5 March 2008).

David, M and C.D. Sutton. 2004. Social research: The basics. London: Sage.

DeVito, JA. 2009. Human communication: The basic course. 11th ed. Boston, MA: Pearson.

Downs, C.W. and M.D. Hazen. 1977. A factor analytic study of communication satisfaction. Journal of business communication 14(3):63-73.

Dozier, D.M., L.A. Grunig and J.E. Grunig. 1995. Manager's guide to excellence in public relations and communication management. Mahwah, $\mathrm{NJ}$ : Lawrence Erlbaum.

Field, A. 2009. Discovering statistics using SPSS: And sex and drugs and rock ' $n$ roll. 3rd ed. Thousand Oaks, Calif: Sage.

Freeman, R.E., A.C. Wicks and B. Parmar. 2004. Stakeholder theory and 'the corporate objective revisited'. Organization Science 15(3):364-369.

Githiora, C. 2002. Sheng: Peer language, Swahili dialect or emerging Creole?. Journal of African cultural studies 15(2):159-181.

Greeff, W.J. 2011. Different salves for different sores: International research remedies for a South African communication context. Communitas 16(2011):113-130.

Grunig, J.E. and L. Hon. 1999. Guidelines for measuring relationships in public relations. (Commission of the Institute for public relations commission on PR measurement and evaluation, Gainesville, Florida). http://www.instituteforpr.org/measureval/rel pi.htm. (accessed 20 February 2011).

Jensen, M.C. 2010. Value maximization, stakeholder theory and the corporate objective function. Journal of applied corporate finance 22(1):32-42.

Keyton, J. 2006. Communication research: Asking questions, finding answers. 2nd ed. Boston, MA: McGraw-Hill.

Le Roux, T and A.M.E. Naudé. 2009. Communicating with diversities: Female employees in the South African platinum-mining industry. Communicare 28(1):24-49. 
Leon, S. 1995. Report of the commission of inquiry into safety and health in the mining industry: Volume 1. Department of Minerals and Energy: Unpublished report.

Lustig, M.W. and J. Koester. 2003. Intercultural competence: Interpersonal communication across cultures. Boston, NJ: Allyn and Bacon.

Meekers, D. 2000. Going underground and going after women: Trends in sexual risk behaviour among gold miners in South Africa. International journal of STD \& Aids 11(1):21-26.

Nyamnjoh, F. 2009. Africa's media: democracy and belonging. In Media and Identity in Africa, ed. K. Njogu and J. Middleton, 62- 75 . Edinburg: Edinburg University Press.

Prinsloo, L. 2010. Mine inspector says South Africa will not compromise on safety. Mining weekly 20 July. http://www.miningweekly.com/article/dmr-invitesconsultative-interpretation-of-new-safety-directive-2010-07-20 (accessed 15 October 2010).

Rao, S. and H. Wasserman. 2007. Global media ethics revisited: A postcolonial critique. Global media and communication 3(1):29-50

Reuters. 2012. Court suspends mine safety stoppage. http://www.fin24.com/Companies/Mining/Court-suspends-mine-safety-stoppage20120313 (accessed 15 March 2012). 